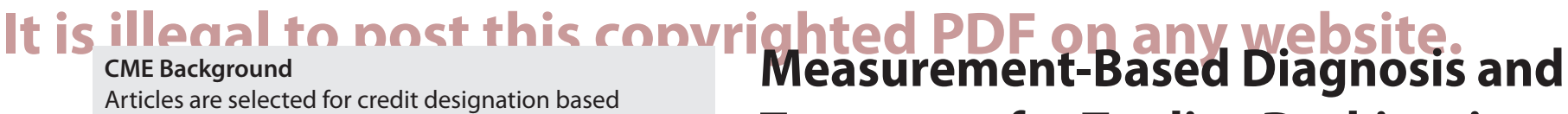 Articles are selected for credit designation based
on an assessment of the educational needs of CME participants, with the purpose of providing readers Treatment for Tardive Dyskinesia
} with a curriculum of CME articles on a variety of topics throughout each volume. Activities are planned using a process that links identified needs with desired results. To obtain credit, read the article, correctly answer the questions in the Posttest, and complete the Evaluation. This ACADEMIC HIGHLIGHTS section of The Journal of Clinical Psychiatry presents the highlights of the teleconference series "Revisiting the Fundamentals of Diagnosing and Treating Tardive Dyskinesia," which was held in March 2021. This activity was prepared and independently developed by the CME Institute of Physicians Postgraduate Press, Inc., and was supported by an educational grant from Neurocrine Biosciences, Inc. The teleconference was chaired by Christoph $\mathbf{U}$. Correll, MD, Zucker School of Medicine at Hofstra/ Northwell, Hempstead, New York; and Charité Universitätsmedizin Berlin, Germany. The faculty member was Leslie Citrome, MD, MPH, New York Medical College, Valhalla.

CME Objective

After studying this article, you should be able to:

- Select appropriate treatment for patients diagnosed with tardive dyskinesia

\section{Accreditation Statement}

The CME Institute of Physicians Postgraduate Press, Inc., is accredited by the Accreditation Council for Continuing Medical Education to provide continuing

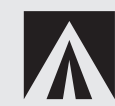
ACCME medical education for physicians.

\section{Release, Expiration, and Review Dates}

This educational activity was published in August 2021 and is eligible for AMA PRA Category 1 Credit $^{\text {TM }}$ through October 31, 2023. The latest review of this material was July 2021.

\section{Financial Disclosure}

All individuals in a position to influence the content of this activity were asked to complete a statement regarding all relevant personal financial relationships between themselves or their spouse/partner and any commercial interest. The CME Institute has resolved any conflicts of interest that were identified. In the past year, Marlene P. Freeman, MD, Editor in Chief, has received research funding from JayMac and Sage; has been a member of the advisory boards for Otsuka, Alkermes, and Sunovion; and has been a member of the Independent Data Safety and Monitoring Committee for Janssen. No member of the CME Institute staff reported any relevant personal financial relationships. Faculty financial disclosure appears on the next page.

J Clin Psychiatry 2021;82(5):NU20016AH2C

To cite: Correll CU, Citrome L. Measurement-based diagnosis and treatment for tardive dyskinesia. J Clin Psychiatry. 2021;82(5):NU20016AH2C.

To share: https://doi.org/10.4088/JCP.NU20016AH2C

(c) Copyright 2021 Physicians Postgraduate Press, Inc.

\author{
Christoph U. Correll, MD, and Leslie Citrome, MD, MPH
}

$T$ ardive dyskinesia (TD) is a drug-induced movement disorder associated with agents that block dopamine receptors, particularly antipsychotics. TD commonly involves involuntary movements of the orofacial muscles and the extremities. As a consequence, TD can be associated with serious physical and psychological impairments. ${ }^{1}$ This report, based on presentations given by Christoph U. Correll, MD, and Leslie Citrome, MD, MPH, will address how to use measurement-based care to diagnose TD and manage TD treatment.

\section{MEASUREMENT-BASED DIAGNOSIS}

Because TD is brought on by the continued use of dopamine receptor blocking agents, such as antipsychotics, many of those at risk are patients with schizophrenia, bipolar disorder, and major depressive disorder. ${ }^{2}$ Dr Citrome explained that treating these psychiatric conditions is critical, and many patients may be unable to avoid long-term treatment with these medications despite the risk of TD.

\section{Tardive Dyskinesia Risk Factors and Prevention}

Dr Citrome stated that 1 of 4 people who have been exposed to dopamine receptor blocking agents have $\mathrm{TD}$, with a higher percentage among those who have been exposed to first-generation antipsychotics (FGAs). ${ }^{3}$ A meta-analysis ${ }^{4}$ of prospective, randomized studies showed that second-generation antipsychotics (SGAs) have a significantly lower risk for TD than FGAs. Among individuals who have been exposed only to SGAs, the incidence rate was about $7 \%{ }^{3}$ Other risk factors for TD include older age, female sex, presence of a mood disorder, higher cumulative exposure to antipsychotics, emergence of parkinsonism or akathisia, treatment with anticholinergic medications, intermittent antipsychotic treatment, and a history of movement disorders. ${ }^{3,5}$

Dr Correll noted the importance of managing the risk of TD by educating patients and caregivers about the risks of and alternatives to antipsychotic medication, as well as early signs of TD to watch for if these medications are taken long-term. Clinicians should confirm and document the indication for use of antipsychotics and use conservative maintenance doses; if allowed by the treated condition, the antipsychotic should be stopped after the shortest necessary time. ${ }^{6}$ Clinicians should also consider the use of SGAs instead of FGAs, especially for patients at high risk for drug-induced movement disorders, including TD.

\section{Screening for Tardive Dyskinesia}

The most recent American Psychiatric Association (APA) guidelines for the treatment of patients with schizophrenia ${ }^{7}$ recommend routine assessments for abnormal movements at a set frequency with a formal examination. The use of a structured 


\section{Itt is illenal to most this cony rit Credit Designation \\ The CME Institute of Physicians Postgraduate Press, Inc., designates this journal-based CME activity for a maximum of 1 AMA PRA Category 1 Credit $^{\mathrm{TM}}$. Physicians should claim only the credit commensurate with the extent of their participation in the activity. \\ Note: The American Nurses Credentialing Center (ANCC) and the American Academy of Physician Assistants (AAPA) accept certificates of participation for educational activities certified for AMA PRA Category 1 Credit $^{\mathrm{TM}}$ from organizations accredited by the ACCME.}

\section{Financial Disclosure}

Dr Correll has received grant/research support and honoraria from AbbVie, Acadia, Alkermes, Allergan, Angelini, Axsome, Gedeon Richter, Gerson Lehrman Group, Indivior, IntraCellular Therapies, Janssen/J\&J, Karuna, LB Pharma, Lundbeck, MedAvante-ProPhase, MedInCell, Medscape, Merck, Mitsubishi Tanabe Pharma, Mylan (Viatris), Neurocrine, Noven, Otsuka, Pfizer, Recordati, Rovi, Servier, Sumitomo Dainippon, Sunovion, Supernus, Takeda, and Teva; has received grant/research support from Janssen and Takeda; is a member of the speakers/advisory boards for Acadia, Alkermes, Allergan, Angelini, Axsome, Gedeon Richter, IntraCellular Therapies, Janssen/J\&J, LB Pharma, Lundbeck, MedlnCell, Merck, Mylan (Viatris), Neurocrine, Noven, Otsuka, Pfizer, Recordati, Rovi, Servier, Sumitomo Dainippon, Sunovion, Supernus, Takeda, and Teva; and is a stock shareholder of LB Pharma. Dr Citrome is a consultant for AbbVie, Acadia, Alkermes, Allergan, Avanir, Axsome, BioXcel, Cadent Therapeutics, Eisai, Impel, Intra-Cellular Therapies, Janssen, Karuna, Lundbeck, Luye, Merck, Neurocrine, Noven, Osmotica, Otsuka, Sage, Shire, Sunovion, Takeda, and Teva; is a member of the speakers/advisory boards for AbbVie, Acadia, Alkermes, Allergan, Eisai, Intra-Cellular Therapies, Janssen, Lundbeck, Merck, Neurocrine, Noven, Otsuka, Sage, Shire, Sunovion, Takeda, Teva; is a stock shareholder of Bristol-Myers, Squibb, Eli Lilly, J\&, Merck, and Pfizer; and has received royalties from Wiley, UpToDate, Springer Healthcare, and Elsevier.

\section{Review Process}

The faculty member(s) agreed to provide a balanced and evidence-based presentation and discussed the topic(s) and CME objective(s) during the planning sessions. The faculty's submitted content was validated by CME Institute staff, and the activity was evaluated for accuracy, use of evidence, and fair balance by the Chair and a peer reviewer who is without conflict of interest.

The opinions expressed herein are those of the faculty and do not necessarily reflect the opinions of the CME provider and publisher or the commercial supporter.

instrument, such as the Abnormal Involuntary Movement Scale (AIMS), is the standard of care. ${ }^{8}$ The AIMS is an observer-rated, 12-item anchored scale. The AIMS itself is not diagnostic (the clinician needs to consider other explanations for any observable abnormal motor movements), but the AIMS can assist in the quantification of the dyskinetic movements, which can be very helpful in documenting a baseline prior to treatment and in providing patient education.

Dr Citrome stated that follow-up assessments using the AIMS should be conducted on a regular basis. Patients who use FGAs should be examined for TD every 6 months, patients who use SGAs and no concomitant FGAs should be examined annually, and patients at high risk for TD should be examined every 3 months if taking FGAs or every 6 months if taking SGAs. ${ }^{2}$

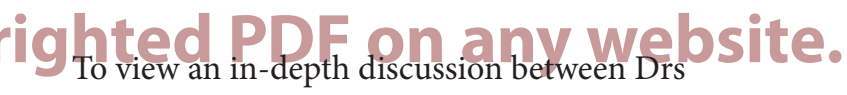

Citrome and Correll on incorporating the AIMS into clinical practice, see the on-demand presentation titled "Diagnostic and Treatment Fundamentals for Tardive Dyskinesia" in this series at cmeinstitute.com.

\section{Differential Diagnosis}

Dr Citrome advised that TD must be differentiated from other movement disorders, particularly druginduced parkinsonism. ${ }^{9}$ Other conditions to consider in the differential diagnosis of TD include spontaneous dyskinesias that can be encountered especially in the elderly, drug-induced dyskinesias caused by other agents, chronic motor tic disorder, Tourette syndrome, autism (stereotypies/stimming), restless legs syndrome, and oral movements from ill-fitting dentures and other dental problems, as well as rarer disorders such as Huntington disease/senile chorea, Meige syndrome, Sydenham chorea, Rett syndrome, and Wilson disease. ${ }^{8,10}$

\section{Patient Perspectives}

Here, in an interview with a representative of Mental Health America (MHA) in January 2021, a patient described the impact of TD on her work:

"I am fluent in American Sign Language (ASL). I went to school to be an interpreter. I'm completely deaf in one ear. I can't be an interpreter anymore because the [TD] movements were so severe I couldn't hold my hands steady long enough to sign in a way where people could typically understand me. I was also certified to be a body piercer. I can't do that anymore because it's a safety hazard. So, I just stopped that. What I was able to do and what I was trained and certified to do, I can no longer do."

\section{Case Practice Question}

Discussion of the best response can be found at the end of the activity.

You are seeing a 34-year-old man with schizophrenia for an intake visit, and you notice that he has abnormal involuntary movements. His clinical history indicates that he has been treated with secondgeneration antipsychotic medication since his diagnosis 12 years ago. He has had parkinsonism and akathisia, for which he received anticholinergic treatment. You decide to perform an Abnormal Involuntary Movement Scale (AIMS) evaluation. Which of the following statements about the AIMS is correct?

a. The AIMS is a diagnostic tool for TD.

b. Given the time required, guidelines do not recommend routine AIMS assessments.

c. The AIMS can assist in measurement-based care and patient education.

d. All of the above

\section{MANAGEMENT APPROACHES}

In his presentation, Dr Correll discussed approaches to managing TD. If possible, clinicians may stop the antipsychotic or reduce the dose. ${ }^{11}$ Increasing the dose could mask TD, but symptoms may re-emerge, leading 
to an SGA, or from one SGA antipsychotic to another, are other interventions to consider. ${ }^{11}$ Dr Correll also highlighted other antidyskinesia agents that may treat $\mathrm{TD}$, all of them with varying degrees of evidence supporting their use, such as vitamin $\mathrm{E}$, vitamin $\mathrm{B}_{6}$, ginkgo biloba, and eicosapentaenoic acid, as well as melatonin, clonazepam, amantadine, donepezil and branched-chain amino acids. ${ }^{11-13}$ Reserpine, an irreversible vesicular monoamine transporter-2 (VMAT2) inhibitor, has too many adverse effects to be considered, and tetrabenazine, an older reversible VMAT2 inhibitor, also is a potential treatment option, but rigorous trials for TD are missing for tetrabenazine. ${ }^{13}$ These treatments are not approved by the US Food and Drug Administration (FDA) to treat TD. ${ }^{11}$

\section{FDA-Approved Treatments}

Dr Correll focused his presentation on 2 FDAapproved agents to treat TD that are recommended by the American Psychiatric Association for the treatment of moderate to severe or disabling TD. ${ }^{7,14}$ Both drugs, deutetrabenazine and valbenazine, are reversible VMAT2 inhibitors that reduce the uptake of biogenic amines, particularly dopamine, into presynaptic vesicles. ${ }^{15}$ Studies have demonstrated that both medications are effective in reducing symptoms of TD acutely and long-term without increased risk of depression or suicidality. ${ }^{14}$ Dr Correll compared key differentiating features of the 2 treatments (Figure 1). ${ }^{16}$ Regardless of which treatment is selected, routine monitoring using the AIMS is highly desirable and is essential to providing measurement-based care. ${ }^{7}$

Deutetrabenazine. Deutetrabenazine is closely related chemically to tetrabenazine but is a deuterated drug-selected hydrogen atoms on the tetrabenazine molecule have been replaced with deuterium (a stable, naturally occurring, nonradioactive isotope of hydrogen). ${ }^{17}$ Deuteration alters the pharmacokinetics of tetrabenazine so that deutetrabenazine has a longer half-life and a lower maximum plasma concentration than tetrabenazine. Increased tolerability and twice-daily dosing (vs 3 doses per day with tetrabenazine) may increase treatment adherence among patients. The initial dose of deutetrabenazine is $6 \mathrm{mg}$ twice per day. ${ }^{14}$ The dose may be increased by increments of $6 \mathrm{mg} / \mathrm{d}$ at weekly intervals with a maximum dose of $24 \mathrm{mg}$ twice a day.

In acute studies, deutetrabenazine was superior to placebo in reducing the severity of dyskinetic movements, measured with the AIMS. ${ }^{14}$ In an open-label extension study, ${ }^{18}$ deutetrabenazine showed improvement in both clinician- and patient-rated Global Impression of Change scale scores. At week 6 , more than $50 \%$ of patients had achieved a "much improved" or "very much improved" outcome. Two-thirds of patients achieved that status by 9 to 12 months, and 3 of 4 patients had done so by the end of 2 years. ${ }^{18}$

Valbenazine. Valbenazine is a highly selective VMAT2 inhibitor. Valbenazine and its principal active metabolite have selective VMAT2 binding, ${ }^{19}$ limiting off-target
Figure 1. Key Features of Deutetrabenazine and Valbenazine

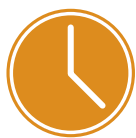

Frequency of administration

Valbenazine: Once daily

Deutetrabenazine: Twice daily

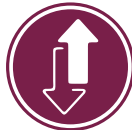

Titration

Valbenazine: Titrate to target dose of $80 \mathrm{mg} / \mathrm{d}$ Deutetrabenazine: Dose to efficacy/tolerability

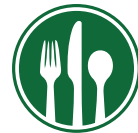

Need for food

Valbenazine: No

Deutetrabenazine: Yes

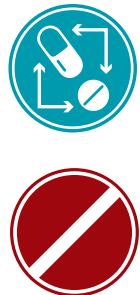

\section{Drug interactions}

Valbenazine: CYP2D6 and CYP3A4 modulators Deutetrabenazine: CYP2D6 modulators

\section{Contraindications}

Valbenazine: Known hypersensitivity to valbenazine or any components of the product

Deutetrabenazine: Hepatic impairment; taking reserpine, monoamine oxidase inhibitors, tetrabenazine, or valbenazine

Reprinted with permission from Citrome and Saklad. ${ }^{16}$

receptor binding. Its half-life is 20 hours, which allows for once-daily dosing. The initial dose for valbenazine is 40 $\mathrm{mg} / \mathrm{d}$, and the maintenance target dose is $80 \mathrm{mg} / \mathrm{d}$, which can be achieved 1 week after the initial dose. ${ }^{14,19}$

In acute studies, valbenazine was superior to placebo in reducing the severity of dyskinetic movements, measured with the AIMS. ${ }^{14}$ An extension study ${ }^{20}$ showed that at the end of one year, 3 of 4 patients receiving the higher dose of $80 \mathrm{mg} / \mathrm{d}$ achieved sustained improvement in both clinician and patient ratings. The lower dose of $40 \mathrm{mg} / \mathrm{d}$ led to similar improvement in $60 \%$ of patients.

\section{Patient Perspectives}

In an interview conducted by MHA in January 2021, a patient described the relief from symptoms that she experienced after receiving effective TD medication:

"I'm currently on [an FDA-approved VMAT 2 inhibitor]. It's taken my movements from a 9 to like a 4 or a 5. [My TD is now] much more tolerable. It's to where people don't notice it as much, that they're not looking at my hands or my legs, focusing in. I don't have vocal tics anymore unless I'm incredibly stressed out."

\section{Patient and Care Partner Communication and Education}

According to Dr Correll, clinical experience suggests that most patients with moderate to severe or disabling TD would be willing to take medication to achieve a reduction in their symptoms, particularly if the medication is well tolerated. ${ }^{21}$ Evidence indicates that the potential benefits of VMAT2 inhibitors for the treatment of TD far outweigh the potential harms. ${ }^{14}$ 
communication with patients and caregivers about risk factors for TD, signs of TD, and treatment options. It is important for clinicians to inquire about and address TD symptoms with each patient and jointly develop treatment goals. The AIMS examination can provide a backdrop for these discussions. Dr Correll offered these example questions: "What is it that you would want the most for yourself (or your loved one) from an effective treatment? What are your goals? How can we measure the outcome to see whether we reach your goals?" Dr Correll also emphasized the importance of setting expectations with patients about medications and their side effects.

\section{Clinical Points}

- Differentiate between TD and other drug-induced movement disorders, particularly drug-induced parkinsonism.

- Use the AIMS examination to screen for and routinely monitor TD, especially when providing treatments intended to decrease the symptoms of TD.

- For patients who have moderate to severe or disabling TD, the APA recommends treatment with FDA-approved VMAT2 inhibitors deutetrabenazine and valbenazine.

- Discuss treatment goals and expectations with patients and care partners.

\section{Discussion of Case Practice Question}

Preferred response is c. The AIMS can assist in measurementbased care and patient education.

The AIMS is not a diagnostic tool, as other medical reasons for the dyskinetic movements need to be ruled out first. This patient is at risk for TD because of long-term antipsychotic treatment, even though he took second-generation medications. Extrapyramidal side effects such as parkinsonism and akathisia are relevant risk factors for TD, and anticholinergic medications for them do not reduce, but potentially further increase, TD risk. The APA guidelines recommend regular use of structured assessments such as the AIMS, in addition to clinical assessments at each visit, to facilitate measurement-based care. The AIMS can be helpful in assisting in the quantification of the dyskinetic movements, which can be useful when documenting a baseline prior to treatment and can aid patient education.

Published online: August 31, 2021.

Disclosure of off-label usage: The chair has determined that, to the best of

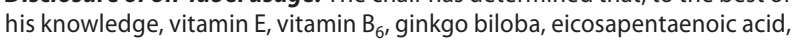
melatonin, clonazepam, amantadine, donepezil, branched-chain amino acids, reserpine, and tetrabenazine are not approved by the US Food and Drug Administration for the treatment of tardive dyskinesia.

\section{REFERENCES}

1. Jain R, Correll CU. Tardive dyskinesia: recognition, patient assessment, and differential diagnosis. J Clin Psychiatry. 2018;79(2):nu17034ah1c.

2. Citrome L. Clinical management of tardive dyskinesia: five steps to success. J Neurol Sci. 2017;383:199-204.

3. Carbon M, Hsieh C-H, Kane JM, et al. Tardive dyskinesia prevalence in the period of second-generation antipsychotic use: a meta-analysis. J Clin Psychiatry. 2017;78(3):e264-e278.

4. Carbon M, Kane JM, Leucht $S$, et al. Tardive dyskinesia risk with first- and second-generation antipsychotics in comparative randomized controlled trials: a meta-analysis. World Psychiatry. 2018;17(3):330-340.

5. Solmi M, Pigato G, Kane JM, et al. Clinical risk factors for the development of tardive dyskinesia. J Neurol Sci. 2018;389:21-27.
6. Caroff SN, Miller DD, Dhopesh V, et al. Is there a rational management strategy for tardive dyskinesia? Curr Psychiatr. 2011;10(10):22-32.

7. Keepers GA, Fochtmann LJ, Anzia JM, et al. The American Psychiatric Association Practice Guideline for the Treatment of Patients With Schizophrenia. 3rd ed. American Psychiatric Association Publishing; 2021.

8. Kane JM, Correll CU, Nierenberg AA, et al. Revisiting the Abnormal Involuntary Movement Scale: proceedings from the Tardive Dyskinesia Assessment Workshop. J Clin Psychiatry. 2018;79(3):17cs11959.

9. Ward KM, Citrome L; Tardive Dyskinesia-Key Differences in Pathophysiology and Clinical Management. Antipsychotic-related movement disorders: drug-induced Parkinsonism vs tardive dyskinesia-key differences in pathophysiology and clinical management. Neurol Ther. 2018;7(2):233-248.

10. Kane JM. Assessing patients for tardive dyskinesia. J Clin Psychiatry. 2017;78(9):e1428.

11. Kaspar R, Ellingrod VL. Strategies for managing drug-induced tardive dyskinesia. Curr Psychiatr. 2014;13(3):44-46.

12. Bhidayasiri R, Fahn S, Weiner WJ, et al; American Academy of Neurology. Evidence-based guideline: treatment of tardive syndromes: report of the Guideline Development Subcommittee of the American Academy of Neurology. Neurology. 2013;81(5):463-469.

13. Kim J, Macmaster E, Schwartz TL. Tardive dyskinesia in patients treated with atypical antipsychotics: case series and brief review of etiologic and treatment considerations. Drugs Context. 2014;3:212259.

14. Solmi M, Pigato G, Kane JM, et al. Treatment of tardive dyskinesia with VMAT-2 inhibitors: a systematic review and meta-analysis of randomized controlled trials. Drug Des Devel Ther. 2018;12:1215-1238.

15. Bernstein Al, Stout KA, Miller GW. The vesicular monoamine transporter 2: an underexplored pharmacological target. Neurochem Int. 2014;73:89-97.

16. Citrome L, Saklad SR. Revisiting tardive dyskinesia: focusing on the basics of identification and treatment. J Clin Psychiatry. 2020;81(2):TV18059AH3C.

17. Citrome L. Breakthrough drugs for the interface between psychiatry and neurology. Int J Clin Pract. 2016;70(4):298-299.

18. Fernandez HH, Stamler D, Davis MD, et al. Long-term safety and efficacy of deutetrabenazine for the treatment of tardive dyskinesia. J Neurol Neurosurg Psychiatry. 2019;90(12):1317-1323.

19. O'Brien CF, Jimenez R, Hauser RA, et al. NBI-98854, a selective monoamine transport inhibitor for the treatment of tardive dyskinesia: a randomized, double-blind, placebo-controlled study. Mov Disord. 2015;30(12):1681-1687.

20. Factor $\mathrm{SA}$, Remington $\mathrm{G}$, Comella $\mathrm{CL}$, et al. The effects of valbenazine in participants with tardive dyskinesia: results of the 1-year KINECT 3 extension study. J Clin Psychiatry. 2017;78(9):1344-1350.

21. Caroff SN, Citrome L, Meyer J, et al. A modified Delphi consensus study of the screening, diagnosis, and treatment of tardive dyskinesia. J Clin Psychiatry. 2020;81(2):19cs12983.

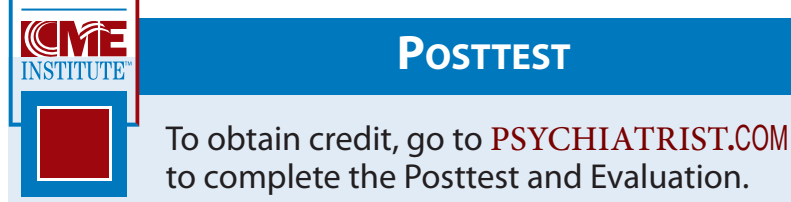

1. For which type(s) of tardive dyskinesia (TD) does the American Psychiatric Association recommend treatment with an approved vesicular monoamine transporter-2 (VMAT2) inhibitor?

a. Emerging symptoms and signs of TD

b. Mild forms of TD

c. Moderate to severe or disabling TD

d. All of the above

2. You are treating a 45 -year-old woman with schizophrenia who has developed moderate TD after 7 years of antipsychotic treatment. When you initiate treatment for TD, which of the following pharmacokinetically driven actions is most appropriate?

a. Prescribe either deutetrabenazine or valbenazine twice daily with food

b. Prescribe either deutetrabenazine or valbenazine once daily with food

c. Prescribe either deutetrabenazine or valbenazine once daily without relationship to food

d. Prescribe deutetrabenazine twice daily with food or valbenazine once daily without relationship to food 